Journal for ImmunoTherapy of Cancer

\title{
TFEB is a master regulator of tumor- associated macrophages in breast cancer
}

\author{
Yong Li, ${ }^{1}$ Johnie Hodge, ${ }^{1}$ Qing Liu, ${ }^{1}$ Junfeng Wang, ${ }^{1}$ Yuzhen Wang, ${ }^{1}$ \\ Trent D Evans, ${ }^{2}$ Diego Altomare, ${ }^{3}$ Yongzhong Yao, ${ }^{4}$ E Angela Murphy, ${ }^{5}$ \\ Babak Razani, ${ }^{2}$ Daping Fan (D) ${ }^{1}$
}

To cite: Li Y, Hodge J, Liu Q, et al. TFEB is a master regulator of tumor-associated macrophages in breast cancer. Journal for ImmunoTherapy of Cancer 2020;8:e000543. doi:10.1136/jitc-2020-000543

- Additional material is published online only. To view please visit the journal online (http://dx.doi.org/10.1136/jitc2020-000543).

$\mathrm{YL}$ and $\mathrm{JH}$ contributed equally.

Accepted 08 May 2020

Check for updates

(c) Author(s) (or their employer(s)) 2020. Re-use permitted under CC BY-NC. No commercial re-use. See rights and permissions. Published by BMJ.

For numbered affiliations see end of article.

Correspondence to

Dr Daping Fan;

daping.fan@uscmed.sc.edu

\section{ABSTRACT}

Background Tumor-associated macrophages (TAMs) play key roles in the development of many malignant solid tumors including breast cancer. They are educated in the tumor microenvironment (TME) to promote tumor growth, metastasis, and therapy resistance. However, the phenotype of TAMs is elusive and how to regulate them for therapeutic purpose remains unclear; therefore, TAM-targeting therapies have not yet achieved clinical success. The purposes of this study were to examine the role of transcription factor EB (TFEB) in regulating TAM gene expression and function and to determine if TFEB activation can halt breast tumor development. Methods Microarrays were used to analyze the gene expression profile of macrophages (MФS) in the context of breast cancer and to examine the impact of TFEB overexpression. Cell culture studies were performed to define the mechanisms by which TFEB affects $M \Phi$ gene expression and function. Mouse studies were carried out to investigate the impact of МФ TFEB deficiency or activation on breast tumor growth. Human cancer genome data were analyzed to reveal the prognostic value of TFEB and its regulated genes.

Results TAM-mimic МФs display a unique gene expression profile, including significant reduction in TFEB expression. TFEB overexpression favorably modulates TAM gene expression through multiple signaling pathways. Specifically, TFEB upregulates suppressor of cytokine signaling 3 (SOCS3) and peroxisome proliferator-activated receptor $\gamma$ (PPAR $\gamma$ ) expression and autophagy/lysosome activities, inhibits NLRP3 (NLR Family Pyrin Domain Containing 3) inflammasome and hypoxia-inducible factor (HIF)-1 $\alpha$ mediated hypoxia response, and thereby suppresses an array of effector molecules in TAMs including arginase 1, interleukin (IL)-10, IL-1及, IL-6 and prostaglandin E2. MФ-specific TFEB deficiency promotes, while activation of TFEB using the natural disaccharide trehalose halts, breast tumor development by modulating TAMs. Analysis of human patient genome database reveals that expression levels of TFEB, SOCS3 and PPAR $\gamma$ are positive prognostic markers, while HIF- $1 \alpha$ is a negative prognostic marker of breast cancer.

Conclusions Our study identifies TFEB as a master regulator of TAMs in breast cancer. TFEB controls TAM gene expression and function through multiple autophagy/ lysosome-dependent and independent pathways.

Therefore, pharmacological activation of TFEB would be a promising therapeutic approach to improve the efficacy of existing treatment including immune therapies for breast cancer by favorably modulating TAM function and the TME.

\section{BACKGROUND}

Tumor-associated macrophages (TAMs) play key roles in the development of many malignant solid tumors including breast cancer. ${ }^{1}$ They are educated in the tumor microenvironment (TME) to aid in tumor growth, metastasis, and therapy resistance. ${ }^{2}$ TAMs have been considered M2-like macrophages (MФs); but they are much more complex than M2 MФs that are typically induced by interleukin (IL)-4/IL-13 signaling. Many signaling pathways in TAMs are triggered by factors within the TME. A broad arsenal of molecules is subsequently produced by TAMs and executes tumor-promoting functions. ${ }^{3}$

During the initiation and development of breast cancer, tumor cells secrete soluble mediators such as colony stimulating factor 1 (CSF1), C-C motif chemokine ligand 2 (CCL2), IL-10, and transforming growth factor $\beta 1$ (TGF $\beta 1$ ), and create a hypoxic and nutrient-depleted milieu. ${ }^{4}$ CSF1 and CCL2 recruit monocytes/macrophages from circulation and promote proliferation of the recruited and mammary tissue resident MФs. ${ }^{5}$ Factors such as IL-10 and TGF $\beta 1$ from cancer cells and tumor stromal cells induce TAMs to secrete molecules such as arginase 1 (Arg1), IL-10, and TGF $\beta 1$, forming paracrine and autocrine loops. Arg1 depletes arginine in the TME, which is essential for $\mathrm{T}$ cell proliferation. ${ }^{6}$ IL-10 directly suppresses $\mathrm{T}$ cell function, ${ }^{7}$ while TGF $\beta 1$ exerts immunosuppression, promotes cancer cell proliferation, and induces epithelial to mesenchymal transition and cancer stem cell generation. ${ }^{8}$

Cytokines such as IL-1 $\beta$ and IL-6, as well as various Toll-like receptor ligands generated within the TME, act on TAMs to induce them to secrete more inflammatory cytokines and growth factors, creating an unresolving chronic inflammatory milieu. ${ }^{910}$ The chronic 
low-grade inflammation results in immune exhaustion and cancer stem cell generation. ${ }^{9}$ Hypoxia in the TME activates hypoxia-induciblefactor $1 \alpha$ (HIF-1 $\alpha)$, which triggers a hypoxia signaling cascade, leading to the production of pro-angiogenic factors such as vascular endothelial growth factor (VEGF) ${ }^{11}$ HIF-1 $\alpha$ signaling also alters lipid metabolism pathways leading to production of PGE2, which is known to cause immunosuppression, angiogenesis, and lymphangiogenesis in breast tumors. ${ }^{12} 13$

Autophagy plays various roles in tumors. On the one hand, cancer cell autophagy may render cancer cells apoptosis resistant. ${ }^{14}$ On the other hand, autophagy and lysosomal activity in MФs may lead to attenuation of inflammation by counteracting the NLRP3 (NLR Family PyrinDomain Containing 3) inflammasome. ${ }^{15}$ Autophagy also may lead to degradation of HIF- $1 \alpha$, de-escalating the hypoxia signaling cascade. ${ }^{16}$

Because TAMs are equipped with a wide range of protumor effector molecules, and MФs are the first line of defense against infection, TAM-targeting cancer treatment strategies via depleting $М \Phi$ s or inhibiting a single type of molecule are either harmful or insufficient. Targeting TAMs at a critical point to keep multiple protumor pathways in check would be more desirable. Therefore, the identification of such a nexus in TAM biology is essential for the future success of TAM-targeting antitumor approaches.

Transcription factor EB (TFEB) controls the expression of genes involved in autophagy and lysosome biogenesis in response to various stimuli. ${ }^{17}$ Under cellular stress conditions, TFEB is translocated from the cytoplasm to the nucleus, and binds to the promoter of the target genes in the Coordinated Lysosomal Enhancement and Regulation (CLEAR) network which are involved in vesicle formation, cargo recognition, lysosome fusion and cargo degradation. ${ }^{18}$ Due to its role in the stress responses, enhancement of TFEB activity has emerged as a potential therapeutic approach for multiple lysosomal and protein aggregation disorders, including atherosclerosis and neurodegenerative diseases. ${ }^{19-21}$

However, the functions of TFEB are not limited to autophagy and lysosome biogenesis. TFEB is activated in MФs during bacterial infection or stimulation with bacterial components, suggesting TFEB might perform evolutionarily conserved defense functions in the innate immune system. ${ }^{22}$ Recently, our laboratory discovered an important role of TFEB in macrophage (MФ) education in breast cancer. ${ }^{23}$ We showed that TFEB is inactivated and downregulated in TAMs by breast cancer cell-derived TGF 31 , leading to an M2-like polarization via signal transducer and activator of transcription 3 (STAT3) activation, independent of the autophagy-lysosome axis. ${ }^{23}$ In this current study, we aim to further examine how deficiency and overexpression of TFEB affects M $\Phi$ gene expression and functions in the context of breast cancer, and to explore if activation of M $\Phi$ TFEB by trehalose, a natural sugar, may serve as a novel therapeutic approach for breast cancer.

\section{MATERIALS AND METHODS}

\section{Animals}

$\mathrm{BALB} / \mathrm{c}$ and C57BL/6 mice (female, 6-8 weeks old) were purchased from Jackson Laboratories (Bar Harbor, Maine, USA). A TFEB ${ }^{\text {flox }}$ mouse line was generated using the ES cells from EUCOMM depository in Europe, which are the same cells used for generation of liver-specific TFEB knockout mice. ${ }^{24}$ LysM-Cre mice were purchased from Jackson Laboratories and crossed with TFEB $^{\text {flox }}$ mice to generate MФ-specific TFEB knockout (MФ-TFEB ${ }^{-/}$)

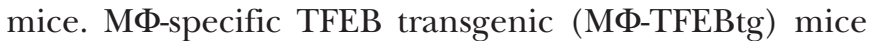
were generated and characterized previously. ${ }^{25}$ Female FVB/N mice were bred with male heterozygous C3(1)/ SV40Tag mice (a gift from Dr Jeffrey Green, National Cancer Institute). All mice were housed in the University of South Carolina or Washington University in St. Louis Animal Research Facilities.

\section{Cell culture}

The 4T1 mouse mammary tumor cell line was obtained from the American Type Culture Collection (ATCC). EO771 cells were maintained in culture as described previously. ${ }^{23}$ Peritoneal $\mathrm{M} \Phi \mathrm{s}$ (pMФs) were isolated from thioglycollate-elicited mice. Bone marrow cells from agematched female wild-type (WT) C57Bl/6, МФ-TFEBtg and $\mathrm{M} \Phi-\mathrm{TFEB}^{-/-}$mice were used to generate bone marrow-derived MФs (BMDMs). Bone marrow cells were seeded in 6-well or 12-well plates for 4 hours, and nonadherent cells were removed, then cultured in complete medium containing 30\% L929 cell-conditioned medium as a source of M-CSF for 7 days. The cells were cultured in high glucose Dulbecco's modified Eagle medium (DMEM; Invitrogen Life Technologies) with $10 \%$ fetal bovine serum (FBS) (Invitrogen) and penicillin/streptomycin at $37^{\circ} \mathrm{C}$ in a humidified $5 \% \mathrm{CO}_{2}$ atmosphere. MФs were polarized according to established protocols. ${ }^{26}$

\section{Tumor conditioned medium}

Tumor conditioned medium (TCM) was prepared by culturing 4T1 or EO771 cells following the procedures described previously. ${ }^{23}$ Briefly, tumor cells were seeded at $5 \times 10^{6}$ cells per $75 \mathrm{~cm}^{2}$ dish and cultured to $90 \%$ confluence. Then, the medium was removed, and the cells were rinsed with serum-free DMEM twice. The medium was then replaced with fresh serum-free DMEM, and the supernatant was collected after 24 hours. The collected TCM was centrifuged to remove cells and filtered through a $0.45 \mu \mathrm{m}$ filter (Corning).

\section{Microarray}

BMDMs from MФ-TFEBtg and WT mice were treated with either serum-free DMEM (control), or serum-free DMEM supplemented with $20 \%$ concentrated EO771 TCM (ECM) for 24 hours. Cells were then rinsed twice with phosphate-buffered saline (PBS) and lysed using $700 \mu \mathrm{L}$ Qiazol. RNA was extracted using the miRNeasy kit (Qiagen). Samples were then further cleaned prior to analysis using the RNeasy MinElute Cleanup Kit (Qiagen) 
protocol. An Agilent 2100 Bioanalyzer was used to determine both the quality and the quantity of the RNA samples. All samples had an RNA integrity number of $>8$. RNA amplification and labeling was performed using the Agilent Low Input Quick Amp labeling kit according to the manufacturer's instructions. RNA was again purified using the Qiagen RNeasy mini kit. Dye incorporation and cRNA yield were also assessed. After labeling, samples were hybridized to SurePrint G3 Mouse GE $8 \times 60 \mathrm{~K}$ Microarrays (Cat. \# G4858A-028005) using a gene expression hybridization kit (Agilent) according to the manufacturer's instructions. A high-resolution Agilent DNA microarray scanner (Cat. \# G2565CA) was used to obtain the raw data and images were saved in TIFF format for subsequent microarray analysis. Data were extracted from these images and background corrected data were uploaded into GeneSpring GX software for analysis. After quantile normalization of the data, differentially expressed genes were identified by using the moderated t-test with Benjamini-Hochberg multiple testing correction. Cut-off values of 0.05 and 2.0 were used for p-value and fold-change, respectively. Unsupervised hierarchical cluster analysis was performed with GeneSpring GX. Gene ontology (GO) analysis was performed with GeneSpring. Ingenuity Pathway Analysis (Qiagen) was performed on these data in order to further investigate the interconnection between differentially expressed genes and their biology. A heatmap was generated using normalized intensities and colored to represent gene expression variations for each individual gene.

\section{In vivo cancer models}

For the tumor growth experiments, female BALB/c or C57BL/6 mice 6-8weeks old, 4T1 or EO771 cells $\left(2 \times 10^{5}\right)$ in $20 \mu \mathrm{L}$ PBS were injected subcutaneously into the mammary fat pads immediately caudal to the fourth pair of nipples. Tumor volume was determined by caliper measurement and using the equation volume $=\left(\right.$ length $\times$ width $\left.{ }^{2}\right) / 2$.

\section{Cell isolation}

Tumors were weighed, cut into small fragments $(<3 \mathrm{~mm})$ and digested in $5 \mathrm{~mL}$ of dissociation solution (RPMI 1640 medium supplemented with $10 \%$ FBS, Collagenase type I $(200 \mathrm{U} / \mathrm{mL})$, and DNase I $(100 \mu \mathrm{g} / \mathrm{mL}))$ for $60 \mathrm{~min}$ at $37^{\circ} \mathrm{C}$. Erythrocytes were lysed with red blood cell lysing buffer (Sigma, St. Louis, Missouri, USA). Cell suspensions were passed through $70 \mu \mathrm{m}$ cell strainers, then washed and resuspended in staining buffer. TAMs (F4/80+) were isolated from tumor suspensions using EasySep PE or FITC Positive Selection Kits (Stem Cell Technologies).

\section{Flow cytometry}

Cell populations in the tumors were analyzed using flow cytometry as previously described. ${ }^{23}$ Briefly, cells were stained with anti-CD3 FITC, anti-CD4 APC or anti-CD8 APC, and anti-CD25 PE (Biolegend) in PBS containing $2 \% \mathrm{FBS}$ for $30 \mathrm{~min}$ at $4^{\circ} \mathrm{C}$. Samples were washed twice with staining buffer and analyzed with flow cytometry using a BD FACS Aria II flow cytometer and CXP software V.2.2. Data were collected for 20,000 live events per sample.

\section{Luciferase reporter assay}

To identify TFEB binding sites in the PPAR $\gamma$ promoter, we searched for the E-box motifs on peroxisomeproliferatoractivated receptor $\gamma(\mathrm{PPAR} \gamma)$ promoter or intron regions and found four E-boxes in the upstream region of mouse TFEB transcription start site. The promoter region containing TFEB binding sites were cloned into pGL3 basic vector to generate three constructs: PPAR $\gamma-1158$ $(-797$ to +361$)$ contains all 4 E-boxes; PPAR $\gamma-891(-530$ to +361$)$ contains $\mathrm{E}$ boxes $2-4$ which are close to each other; and PPAR $\gamma-464(-103$ to +361$)$ does not contain any E-boxes. RAW264.7 cells were seeded in triplicate in 12-well plates and allowed to settle for 12 hours, then transfected with one of the above pGL3-PPAR $\gamma$ promoter plasmids or empty pGL3 plasmids and pRL-CMV (internal control) as well as either lentiviral TFEB (PWPI-TFEB) or control lentiviral vector (PWPI). The luciferase of both firefly and Renilla signals were measured using Dual Luciferase Reporter assay kits (Promega, Madison, Wisconsin, USA). Luciferase activity was normalized to Renilla activity to control for transfection efficiency. The level of luciferase activity of the empty vector and in the absence of TFEB (PWPI) was defined as ' 1 '. Fold activation was estimated according to this level of activity.

\section{Quantitative real-time PCR}

Total RNA was isolated and purified using Qiagen RNeasy Kits (Qiagen). RNA ( $2 \mu \mathrm{g})$ was then reverse-transcribed using iScript cDNA Synthesis Kit (Bio-Rad). Quantitative real-time PCR (qPCR) was conducted on a CFX96 system (Bio-Rad) using iQ SYBR Green Supermix (Bio-Rad). All primers used for qPCR analysis were synthesized by Integrated DNA Technologies. All assays were conducted following the manufacturer's instructions. The relative amount of target mRNA was determined using the comparative threshold (Ct) method by normalizing target mRNA Ct values to those of $18 \mathrm{~S}$ RNA. PCR thermal cycling conditions were $3 \mathrm{~min}$ at $95^{\circ} \mathrm{C}$, and 40 cycles of $15 \mathrm{~s}$ at $95^{\circ} \mathrm{C}$ and $58 \mathrm{~s}$ at $60^{\circ} \mathrm{C}$. Samples were run in triplicate. The primer sequences are listed in online supplementary table S1.

\section{Western blot analysis}

Whole cell lysate was prepared using RIPA buffer (Pierce) supplemented with protease inhibitor cocktail and phosphatase inhibitor cocktail (Sigma). The protein concentrations were determined using the BCA protein assay kit (Pierce, Rockford, Illinois, USA). Samples were diluted in $2 \times$ Laemmli buffer (Bio-Rad) and boiled for $10 \mathrm{~min}$. Proteins $(20 \mu \mathrm{g})$ were separated in $10 \%$ SDSpolyacrylamide gel electrophoresis precast gels (BioRad) and transferred onto nitrocellulose membranes (Bio-Rad). Non-specific binding sites on the membranes were blocked with $5 \%$ non-fat milk in phosphate buffered 
saline with tween 20 (PBST). Membranes were first probed with TFEB (1:2000; Bethyl Laboratories), PPAR $\gamma$ (1:1000), NLRP3, p-p65, p65, Lamp1, Hifl $\alpha$, MIF, cytosolic phospholipases A2 (cPLA2), inducible nitric oxide synthase (iNOS), arginase 1 (Arg1), or $\beta$-actin (1:1000; Sigma) antibodies, followed by goat anti-rabbit or antimouse secondary antibody conjugated with horseradish peroxidase (Millipore). Protein detection was conducted using Pierce ECL Substrate (Pierce).

\section{Transcriptomic data retrieval and survival analysis}

The breast cancer patient survival data were obtained from The Cancer Genome Atlas (TCGA) database and Kaplan-Meier plotter database (www.kmplot.com). ${ }^{27}$ Based on the best expression cut-off value (FPKM) of each gene, patients were classified into two groups, association between survival rate and gene expression was examined, or the HR was calculated. Survival curves were estimated with the Kaplan-Meier method and compared using the log-rank test.

\section{Immunofluorescence staining}

For breast tumor tissues from patients, deidentified formalin-fixed paraffin embedded tissues were collected from mastectomy surgery with ethical approval by Nanjing Drum Tower Hospital in 2015. Sections were cut $(4 \mu \mathrm{m}$ thick), transferred to a warm water bath, and placed on a glass slide. The following immunofluorescence staining test was examined by the corresponding anti-human antibodies: TFEB (1:300, Invitrogen) and CD68 (1:300, Abcam).

\section{Statistical analysis}

Data were presented as mean $\pm \mathrm{SD}$ or SEM as indicated. Statistical significance was calculated using the Student's t-test (two group comparison) or one-way analysis of variance (ANOVA) followed by post hoc Dunnett's test (multigroup comparison) using the GraphPad Prism V.6.0. For the TCGA database analysis, $p$ values were calculated for the comparison of the 5-year survival rates between the two groups. For the Kaplan-Meier plotter database, univariate Cox proportional hazard modeling of the genes was used to determine their independent impact on patients' survival, and to estimate the corresponding HR, setting low expression as the reference group; HRs and $95 \% \mathrm{CI}$, as well as the $\mathrm{p}$ values, were reported. $\mathrm{P}<0.05$ was considered significant.

\section{RESULTS}

Breast cancer cell conditioned medium treatment results in a unique gene expression profile in MФs

TAMs are often considered to adopt an M2 phenotype, which is typically induced by IL-4 or IL-13. However, the TME is a complex milieu; in addition to IL-4 and IL-13, many other factors, including hypoxia, apoptotic cancer cells and their debris, growth factors, and proinflammatory or anti-inflammatory cytokines secreted from cancer cells and stromal cells, act on TAMs. Therefore, the phenotype of TAMs is much more complex than that of typical M2 MФs. Breast cancer EO771 cell conditioned medium (ECM) was used to treat BMDMs to mimic TAMs, and a microarray was performed to compare the gene expression profiles between those TAM-mimic cells and control MФs. The results are summarized in a heatmap; we also plot a heatmap to compare the expression of the same set of genes in control MФs, M1 MФs (induced by LPS+IFN $\gamma$ ), and M2 MФs (induced by IL-4) using the data we obtained from a previous microarray, ${ }^{28}$ in order to visualize the gene expression differences between TAM-mimic cells and M1 or M2 MФs (figure 1A). First, we confirmed that like in IL-4 induced M2 MФs, some typical M2 markers, including Arg1, IL-10, and TGF $\beta 1$, are increased in ECMtreated MФs. However, unlike in M2 MФs, the expression of many M1 markers, such as IL-1 $\beta$, IL-6, tumor necrosis factor $\alpha(\mathrm{TNF} \alpha)$, iNOS and NLRP3, also are significantly increased in ECM-treated MФs. Additionally, more resembling M1 than M2 MФs, ECM-treated MФs have elevated expression of HIF-1 $\alpha$, macrophage migration inhibitory factor (MIF), cPLA2, and cyclo-oxygenase-2 (COX2). COX2 catalyzes the production of prostaglandin E2 (PGE2), a key tumor-promoting and immune-suppressive metabolite in the TME. Also, unlike M2 MФs but more like M1 MФs, ECM-treated MФs have reduced expression of TFEB and PPAR $\gamma$. PPAR $\gamma$ controls several inflammatory response and cellular metabolism pathways. Using western blot analysis, we confirmed that TFEB and PPAR $\gamma$ are increased in M2 MФs, but reduced in ECM-treated MФs; while NLRP3 is reduced in M2 MФs, but increased in ECM-treated МФs (figure 1B).

\section{MФ TFEB ablation promotes breast tumor growth}

We previously showed that TFEB overexpression in МФs attenuated orthotopic breast tumor growth, while injection of MФs with lentiviral knockdown of TFEB accelerated breast tumor growth. ${ }^{23}$ To further examine the function of MФ TFEB in breast tumors, we aimed to test if TFEB ablation in MФs promotes breast tumor growth via conferring TAMs with a more tumor-promoting phenotype. We therefore generated MФ-specific TFEB knockout

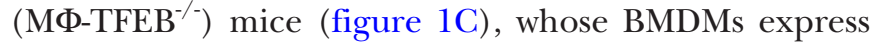
TFEB at only 25\% level of WT control (figure 1D,E). We generated bone marrow chimeric mice by transplanting bone marrow cells from $\mathrm{M} \Phi-\mathrm{TFEB}^{-/-}$mice or Cre-littermates to lethally irradiated WT $\mathrm{C} 57 \mathrm{Bl} / 6$ mice using our standard protocol. ${ }^{23}$ We implanted EO771 cells into mammary fat pads of the mice 4 weeks after the bone marrow transplantation. Tumor growth was monitored for 35 days, showing TFEB ablation in MФs promoted tumor growth (figure 1F). At sacrifice, the weight of tumors from mice transplanted with bone marrow cells from $\mathrm{M} \Phi-\mathrm{TFEB}^{-/-}$mice was significantly increased compared with that of control mice (figure 1G). Single cell suspensions were obtained from tumors, and flow cytometry analysis showed that tumors in $\mathrm{M} \Phi-\mathrm{TFEB}^{-/}$bone marrow recipient mice contained similar percentages of total 

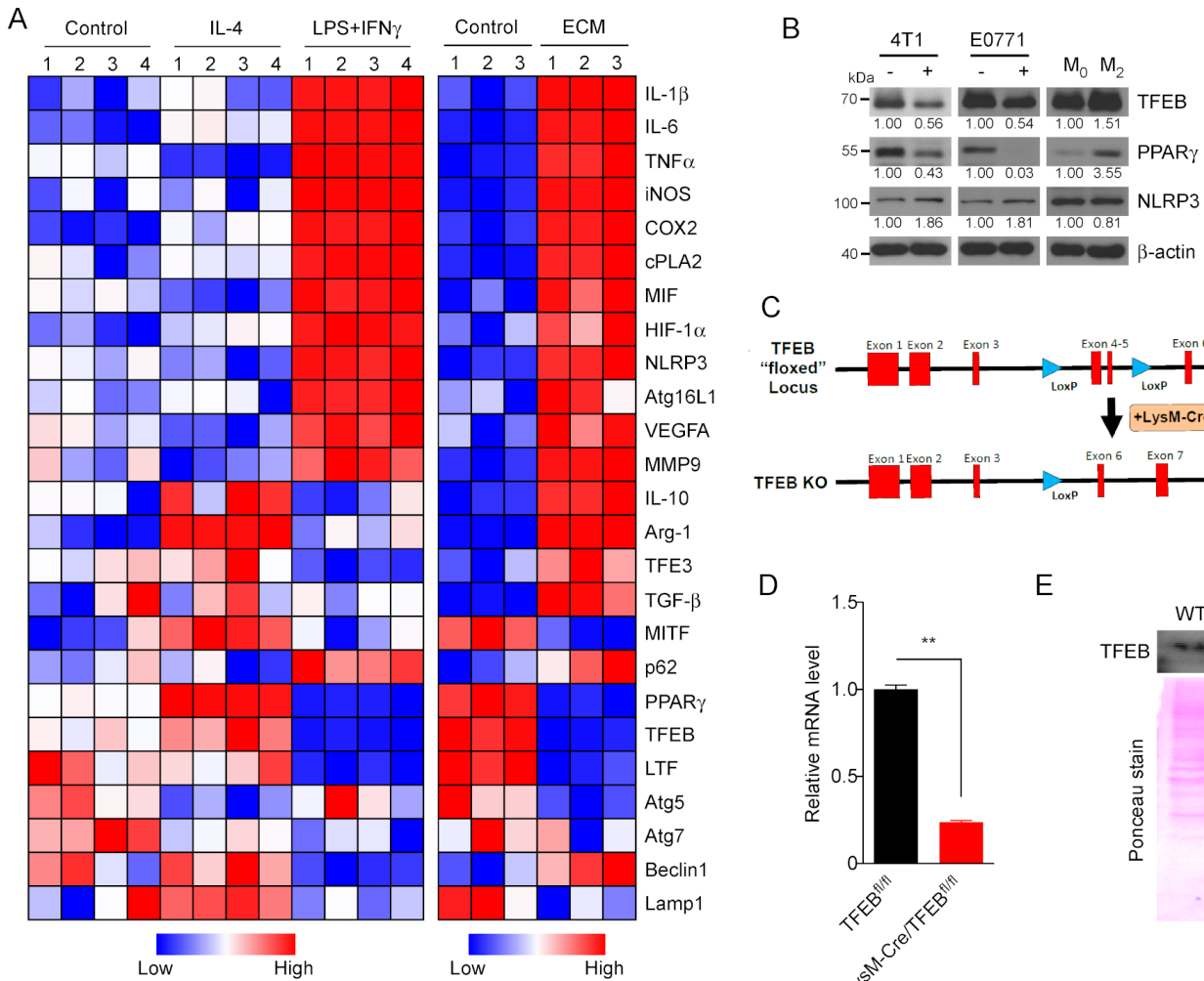

C

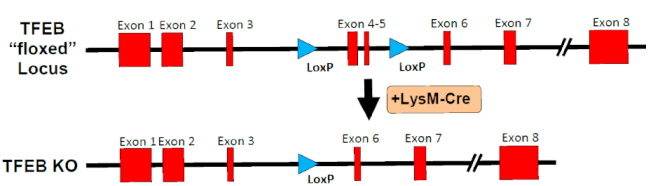

D

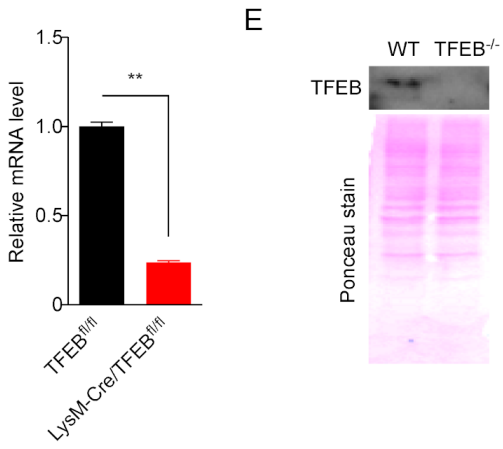

F

G

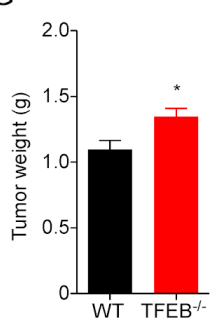

$\mathrm{H}$
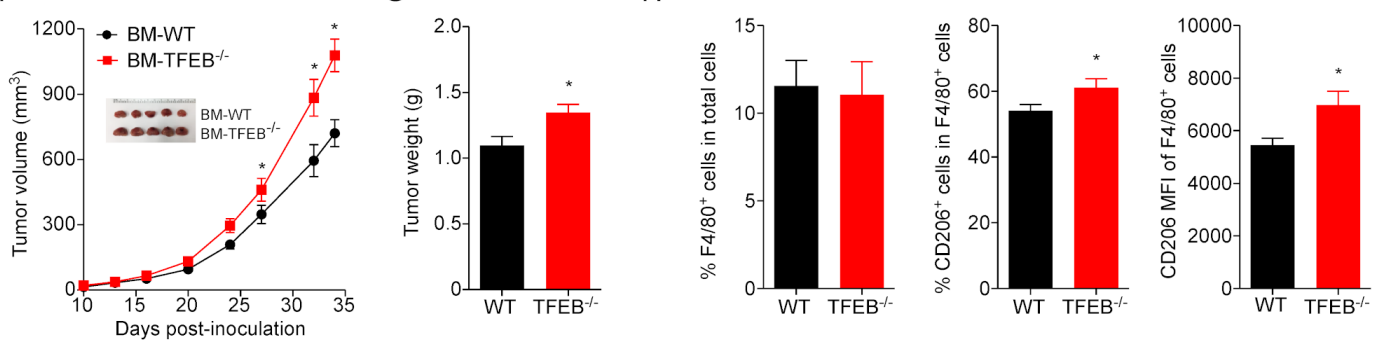

I
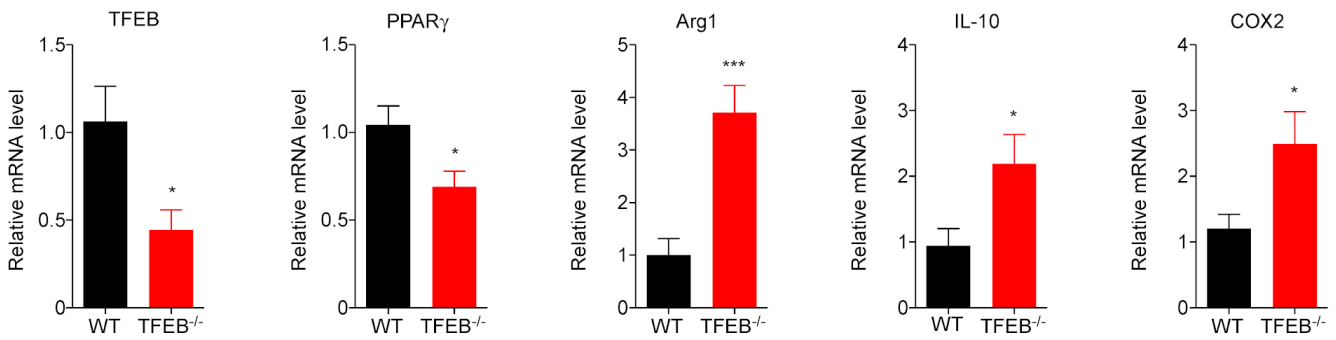

Figure 1 Downregulation of TFEB renders macrophages (MФs) tumor-promoting. (A) Clustering of gene expression profiles of individual runs for all common genes upregulated or downregulated by IL-4, LPS+IFN $\gamma$, or EO771 cell conditioned medium (ECM) are depicted as heatmap matrices according to the color scale shown at the bottom. Each group has four or three biological replicates. The microarray data have been deposited in Gene Expression Omnibus (GEO) database (accession numbers GSE73311 and GSE139554). (B) Western blot analysis of TFEB, PPAR $\gamma$, and NLRP3 proteins in mouse peritoneal MФs treated with or without 4T1 or ECM for 24 hours, and in IL-4 induced M2 MФs with a comparison to non-treated M0 cells. (C) The strategy to generate MФ-specific TFEB knockout mice. A map of the TFEB locus shows the location of LoxP sites and deletion of Exons 4-5 on exposure to LysM-Cre. (D-E) Quantitative real-time PCR (qPCR)(D) and Western blot (E) of peritoneal MФs derived from control $\left(\right.$ TFEB $^{\mathrm{fl} / \mathrm{fl}}$ ) and MФ-TFEB-KO mice. Ponceau stain of total protein is shown as loading control. In qPCR data, data are shown as mean \pm SEM, $n=3$, ${ }^{* *} \mathrm{p}<0.01$, two-tailed Student's t-test. (F) Tumor growth curve in C57BI/6 mice transplanted with bone marrow cells from TFEB $^{\text {fl/fl }}(B M-W T)$ or MФ-TFEB-KO $\left(B M-\right.$ TFEB $\left.^{-/}\right)$mice, with representative tumors shown as inserts. (G) Mice were sacrificed, and the tumor weight of each mouse was measured on day 35 after tumor cell inoculation. $(\mathrm{H})$ Flow cytometry analysis of MФs in the cells isolated from the resected orthotopic breast tumors. (I) Gene expression in MФs isolated from the breast tumors was analyzed by qPCR. Data are shown as mean $\pm S E M, n=3,{ }^{*} p<0.05$, ${ }^{\star *} \mathrm{p}<0.01,{ }^{* *} \mathrm{p}<0.001$; two-tailed Student's t-test. Arg1, arginase 1; COX2, cyclo-oxygenase-2; cPLA2, cytosolic phospholipases A2; HIF-1 $\alpha$, hypoxia-inducible factor $1 \alpha$; IL, interleukin; NLRP3, NLR Family Pyrin Domain Containing 3; PPAR $\gamma$, peroxisome proliferator-activated receptor $\gamma$; TFEB, transcription factor EB; TNF $\alpha$, necrosis tumor factor $\alpha$; VEGFA, vascular endothelial growth factor A; WT, wild type. 
F4 $/ 80^{+}$MФs or $\mathrm{CD}^{+} \mathrm{T}$ cells, but more CD206 ${ }^{+} \mathrm{M} \Phi$ s and $\mathrm{CD}^{+} / \mathrm{CD}^{+} \mathrm{T}$ lymphocytes (figure $1 \mathrm{H}$ and online supplementary figure S1). MФs were isolated from the tumors, and gene expression was analyzed using qPCR. The result shows that TAMs from the tumors in MФ-TFEB ${ }^{-/}$bone marrow recipient mice had lower expression levels of TFEB $(\sim 50 \%)$ and PPAR $\gamma(\sim 70 \%)$, but higher levels of Arg1 ( 3.7-fold), IL-10 ( 2.2-fold), and COX2 ( 2.5-fold) (figure 1I), suggesting TFEB ablation exaggerated the tumor-promoting phenotype of TAMs in breast tumors.

\section{TFEB overexpression modifies MФ gene expression in response to TCM treatment}

With the eventual goal of developing strategies to increase TFEB expression or activate TFEB in TAMs to halt breast tumor development, we next examined how TFEB overexpression may ameliorate the aberrant gene expression profile in TAMs. We have generated MФ-TFEBtg mice in our previous studies. ${ }^{2123}$ BMDMs from WT or MФ-TFEBtg mice were treated with ECM and a microarray analysis was performed. A heatmap shows that 148 genes were downregulated, and 140 genes were upregulated in ECMtreated TFEBtg MФs compared with ECM-treated WT MФs (online supplementary figure S2A). Most significantly affected pathways relevant to $\mathrm{M} \Phi$ functioning in inflammation and cancer were determined by Ingenuity IPA canonical pathway analyses. It was found that among the most significantly suppressed pathways in TFEBtg cells includes HIF-1 $\alpha$ signaling, IL-10 signaling, IL-6 signaling, and VEGF signaling pathways (online supplementary figure S2B). The differences between WT and TFEBtg MФs in the expression levels of the genes shown in figure $1 \mathrm{~A}$ were also examined. It was shown that TFEB overexpression significantly ameliorates the tumor-promoting gene expression profile in TAM-like $\mathrm{M} \Phi$ s (figure 2A). The microarray results were confirmed by qPCR (figure 2B). These results suggest that TFEB overexpression may alter the TAM-like M $\Phi$ phenotype by (1) suppressing typical M2 MФ markers such as Arg1, IL-10, MMP9, and VEGFA; these molecules are known to cause immunosuppression and tumor angiogenesis; (2) increasing the expression of genes involved in autophagy and lysosome biogenesis, such as LC3, Lamp1, P62, and ATG5; (3) enhancing PPAR $\gamma$ and inhibiting the NLRP3/ IL-1 $\beta /$ IL-6 inflammatory axis; and (4) suppressing the PGE2 production axis HIF1 $\alpha /$ MIF/cPLA2/COX2. We examined in our microarray data set if TFEB overexpression affected the expression of genes related antigen presenting function of $M \Phi$ s when they are treated with TCM, such as $\beta 2$-microglobulin, cathepsin E, and Tap1. We found no such genes were differentially expressed in WT or TFEBtg MФs, suggesting that TFEB expression levels may not affect $\mathrm{M} \Phi$ s antigen presentation.

Western blot was performed to confirm some of the gene expression results using WT, TFEBtg and $\mathrm{TFEB}^{-/}$ BMDMs. The results show that, in ECM-treated BMDMs, TFEB overexpression increased the protein levels of PPAR $\gamma$ and Lamp1 but decreased the phosphorylation of p65 and the protein levels of NLRP3, HIF-1 $\alpha$, MIF, cPLA2, and Arg1, while TFEB knockout had the opposite effects on these proteins (figure 2C). We further examined the impact of TFEB overexpression on the production of three direct M $\Phi$ effector molecules IL-1 $\beta$, IL- 6 and PGE2. We treated WT and TFEBtg BMDMs with ECM for 24 hours, then the cells were rinsed and cultured in fresh, serum-free DMEM for a further 16 hours before the medium was collected for ELISA measurement of IL-1 $\beta$ and IL-6, and colorimetric measurement of PGE2. The data show that TFEB overexpression significantly suppressed the elevated levels of IL-1 $\beta$, IL-6 and PGE2 in ECM-treated BMDMs (figure 2D).

\section{TFEB directly regulates PPAR $\gamma$ expression in TAMs}

TFEB binds to a short consensus sequence CANNTG called the E-box on target gene promoters or intron regions to control gene transcription. ${ }^{29}$ It has been reported that TFEB transcriptionally regulates PPAR $\gamma$ expression in human adipocytes. ${ }^{30}$ To test if TFEB directly regulates PPAR $\gamma$ transcription in mouse MФs, we performed a promoter assay. As shown in figure 3A, TFEB overexpression increased luciferase activity in the cells transfected with E-box containing plasmids PPAR $\gamma$ 1158 and PPAR $\gamma-891$, suggesting that the first and at least one of 2-4 E-boxes are directly involved in TFEB binding.

To provide further evidence that PPAR $\gamma$ is directly involved in mediating the effects of TFEB on TAMs, the PPAR $\gamma$ antagonist GW9662 was used to inhibit its activity in BMDMs. WT and TFEBtg BMDMs were treated with ECM with or without $10 \mu \mathrm{M}$ GW9662 for 24 hours (figure 3B,C and online supplementary figure S3). Both mRNA and protein levels of TFEB in BMDMs were not altered by PPAR $\gamma$ inhibition, and PPAR $\gamma$ inhibition did not change Beclin 1 protein levels in BMDMs. Interestingly, while PPAR $\gamma$ mRNA expression was not changed by GW9662, its protein levels were remarkably reduced by GW9662 in ECM-treated TFEBtg BMDMs to a level comparable with ECM-treated WT BMDMs (both almost undetectable). The mechanism by which GW9662 reduces PPAR $\gamma$ protein in BMDMs is unknown. Importantly, PPAR $\gamma$ inhibition increased p65 phosphorylation and the expression of NLRP3, IL-1 $\beta$, HIF-1 $\alpha$, and cPLA2 in both ECM-treated WT and TFEBtg BMDMs. The differences between ECMtreated WT and TFEBtg BMDMs in p65 phosphorylation and expression of NLRP3 (mRNA and protein), IL-1 $\beta$ (mRNA), HIF-1 $\alpha$ (protein), and cPLA2 (protein) were diminished, but the differences in HIF- $1 \alpha$ and cPLA2 mRNA levels were not decreased. These results suggest that PPAR $\gamma$ partially mediates the suppressive effects of TFEB on NF- $\mathrm{KB}$ activation and NLRP3 and HIF- $1 \alpha$ upregulation in ECM-treated BMDMs. Taken together, these data demonstrate that TFEB directly regulates PPAR $\gamma$ transcription and partially through this mechanism, TFEB modulates NLRP3/IL-1 $\beta$ and HIF1 $\alpha /$ cPLA2 pathways in MФs.

TFEB's regulation of MФs is partially dependent on autophagy TFEB is a key regulator of autophagy and lysosome biogenesis,${ }^{20}$ and autophagy and associated lysosomal 
A

\begin{tabular}{|c|c|c|c|}
\hline Gene & Function & $\begin{array}{l}\text { Relative } \\
\text { expression }\end{array}$ & $p$ \\
\hline Arg1 & M2 marker & 0.018 & $<0.0001$ \\
\hline MMP9 & $\begin{array}{l}\text { Invasion, } \\
\text { angiogenesis }\end{array}$ & 0.11 & $<0.0001$ \\
\hline IL-10 & Anti-inflammation & 0.11 & $<0.0001$ \\
\hline IL-6 & Inflammation & 0.15 & $<0.0001$ \\
\hline $\cos 2$ & Inflammation & 0.26 & $<0.0001$ \\
\hline MIF & Inflammation & 0.41 & 0.0002 \\
\hline NLRP3 & Inflammasome & 0.51 & 0.0006 \\
\hline iNOS & M1 marker & 0.52 & $<0.0001$ \\
\hline VEGFA & Angiogenesis & 0.58 & 0.003 \\
\hline IL-1B & Inflammation & 0.63 & 0.0006 \\
\hline cPLA2 & Inflammation & 0.65 & $<0.0001$ \\
\hline HIF-1a & Angiogenesis & 0.71 & 0.19 \\
\hline TGFß1 & Tumor growth & 0.86 & 0.12 \\
\hline TNFa & Inflammation & 0.88 & 0.075 \\
\hline Beclin 1 & Autophagy & 0.89 & 0.37 \\
\hline LC3 & Autophagy & 0.92 & 0.49 \\
\hline TFE3 & MiT/TFE family & 0.94 & 0.52 \\
\hline Lamp1 & Lysosome & 0.95 & 0.33 \\
\hline Atg7 & Autophagy & 1.01 & 0.83 \\
\hline TLR4 & Inflammation & 1.04 & 0.53 \\
\hline LTF & Anti-cancer & 1.10 & 0.63 \\
\hline Atg16L1 & Autophagy & 1.16 & 0.40 \\
\hline Atg 5 & Autophagy & 1.17 & 0.054 \\
\hline p62 & $\begin{array}{l}\text { Autophagy/ } \\
\text { Lysosome }\end{array}$ & 1.29 & 0.012 \\
\hline MITF & MiT/TFE family & 1.33 & 0.023 \\
\hline PPAR $\gamma$ & Anti-inflammation & 2.64 & 0.003 \\
\hline
\end{tabular}

C

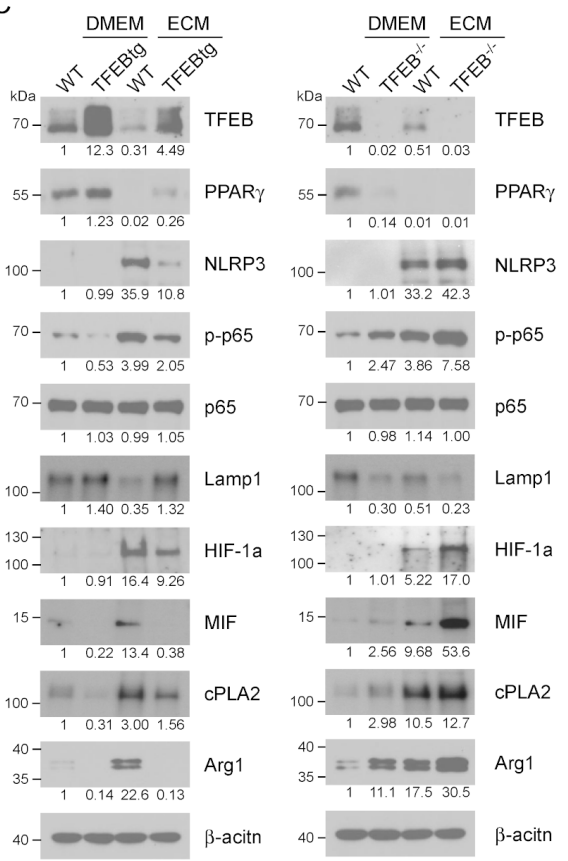

B
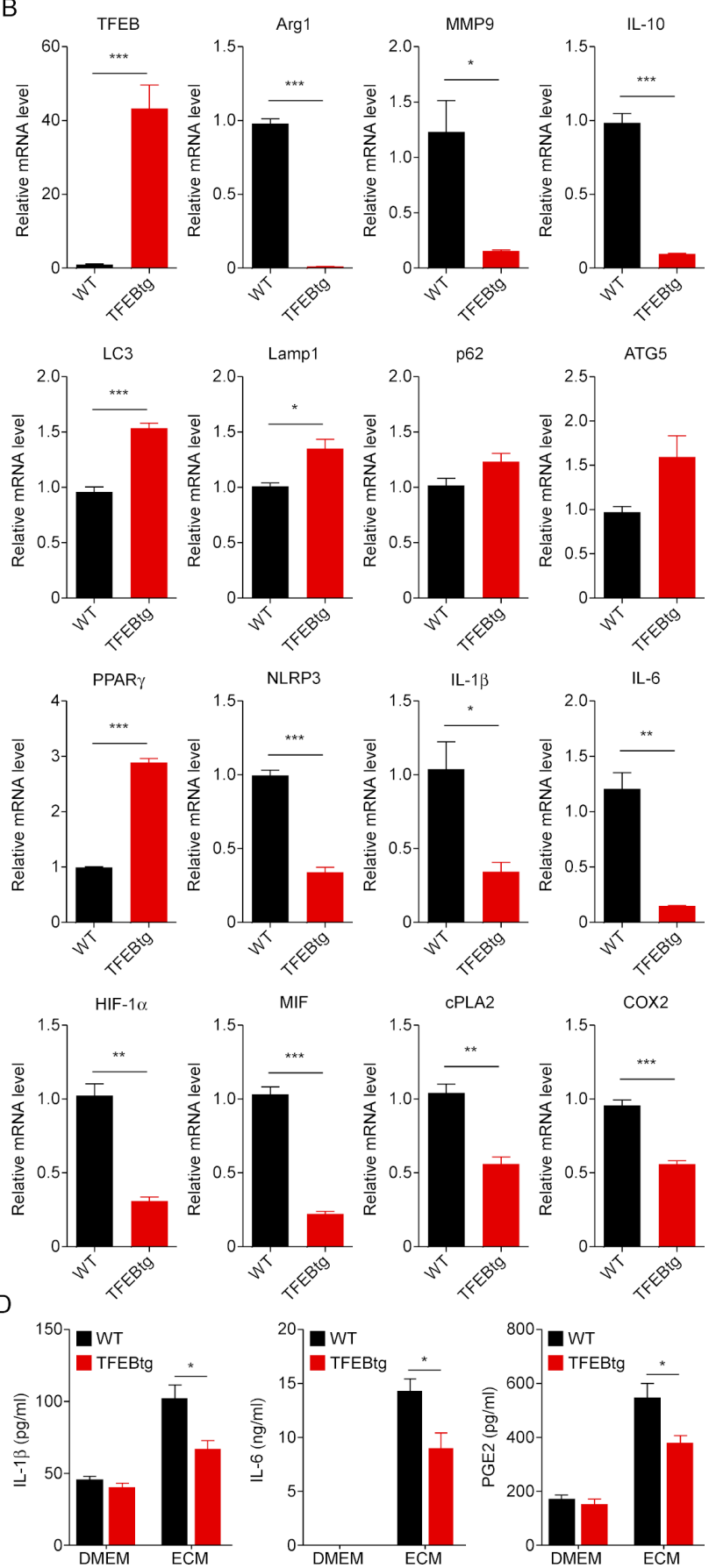

Figure 2 Transcriptionfactor EB (TFEB) overexpression alters the gene expression profile in macrophages (MФs). (A) Bone

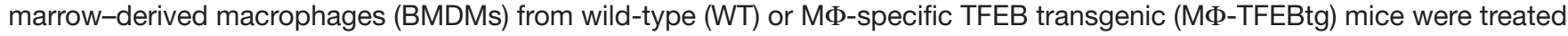
with Dulbecco's modified Eagle medium (DMEM) or EO771 cell conditioned medium (ECM) for 24 hours; microarray was performed to analyze their gene expression profiles. A heatmap of genes differentially expressed in WT or TFEBtg is shown in online supplementary figure S2. A list of key genes that play roles in tumor-associated macrophage (TAM) functions is shown; relative expression means the ratio of the gene expression levels in TFEBtg MФs compared with those in WT MФs. The microarray data have been deposited in Gene Expression Omnibus (GEO) database (accession number GSE139554). (B) Quantitativereal-time PCR (qPCR) confirmation of the microarray data for the key genes. $n=3,{ }^{*} p<0.05 ;{ }^{* *} p<0.01 ;{ }^{* * *} p<0.001$; two-tailed Student's t-test. (C) Western blot analysis of indicated proteins in BMDMs from WT, MФ-TFEBtg or MФ-TFEB ${ }^{-/}$mice. The cells were treated with ECM for 24 hours. The number under a band indicates the relative intensity of the protein to $\beta$-actin (the relative intensity of the first band is set as 1). A representative blot is shown. (D) BMDMs from WT and TFEBtg MФs were treated with DMEM or ECM for 24 hours; then the medium was replaced with fresh DMEM, and the conditioned medium was collected 16 hours later for analysis for IL-1 $\beta$, IL-6 and prostaglandin E2 contents. $n=5$, ${ }^{*} p<0.05$; two-tailed Student's t-test. Arg1, arginase 1; COX2, cyclo-oxygenase-2; cPLA2, cytosolic phospholipases A2; HIF-1 $\alpha$, hypoxia-inducible factor 1 $\alpha$; IL, interleukin; NLRP3, NLR Family Pyrin Domain Containing 3; PPAR $\gamma$, peroxisome proliferator-activated receptor $\gamma$; TNF $\alpha$, necrosis tumor factor $\alpha$. 
B

A

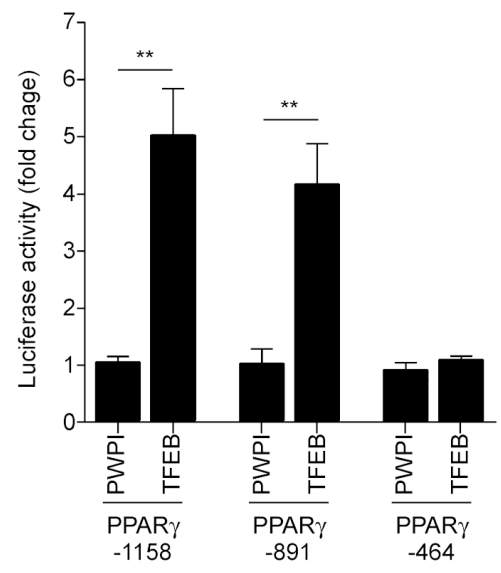

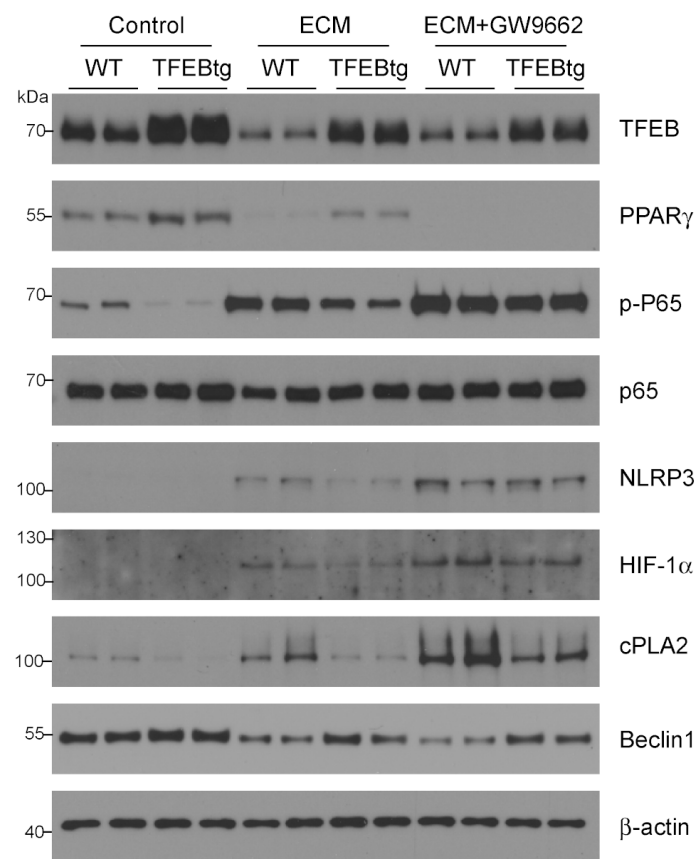

C
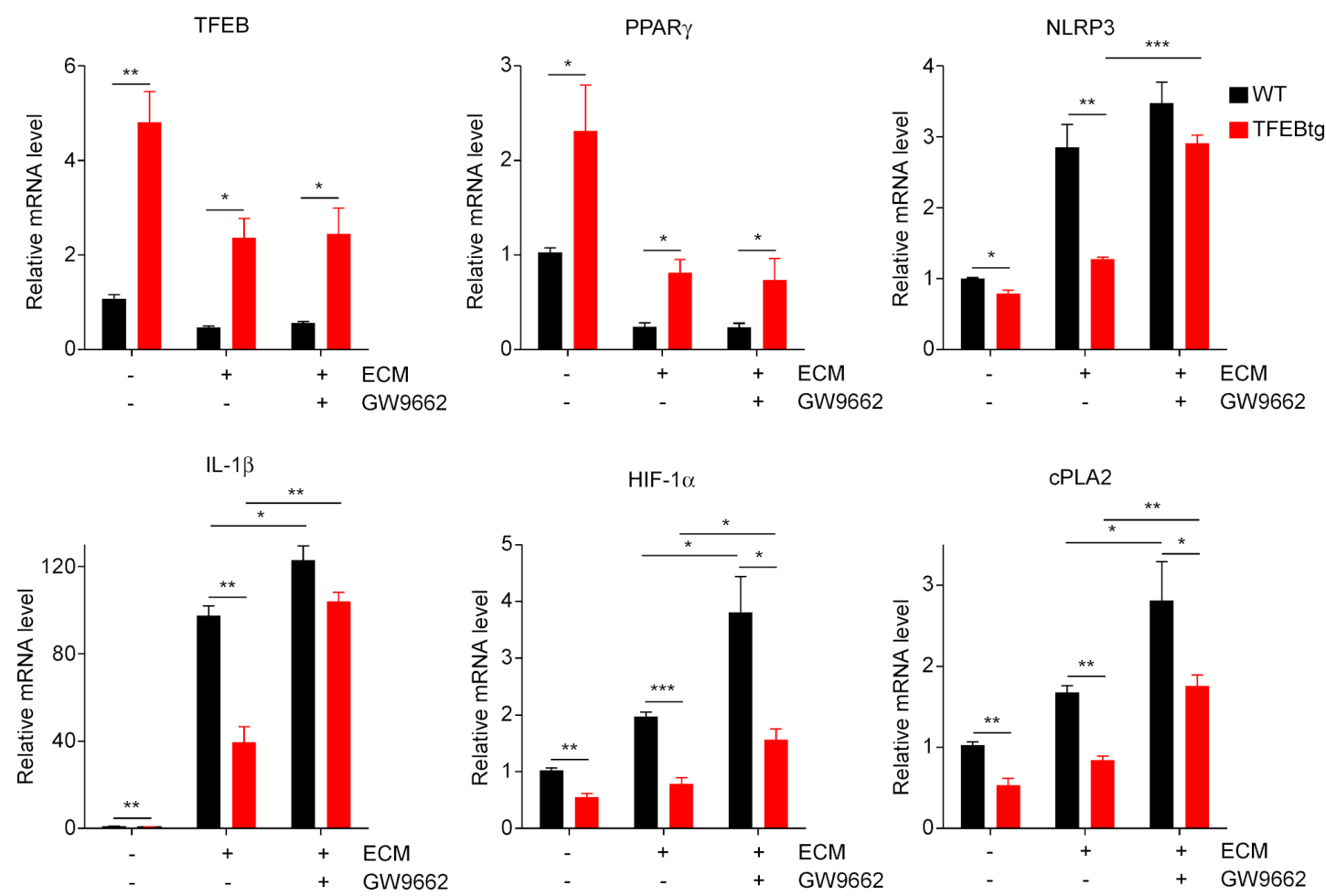

Figure 3 PPAR $\gamma$ mediates part of the effects of TFEB in macrophages (MФs). (A) Luciferase promoter activity assay of the binding of TFEB to PPAR $\gamma$ promoter regions; ${ }^{* *} p<0.01$. (B) Western blot analysis of the indicated protein levels in wild-type (WT) or TFEB transgenic (TFEBtg) bone marrow-derived MФs (BMDMs) treated with EO771 cell conditioned medium (ECM) with or without GW9662, a PPAR $\gamma$ antagonist. The quantification is shown in online supplementary figure S3. (C) Quantitative realtime PCR analysis of expression of the indicated genes in WT and TFEBtg BMDMs treated with ECM with or without GW9662. $n=3$; ${ }^{*} p<0.05,{ }^{* *} p<0.01,{ }^{* * *} p<0.001$; two-tailed Student's t-test. Arg1, arginase 1; COX2, cyclo-oxygenase-2; cPLA2, cytosolic phospholipases A2; HIF-1 $\alpha$, hypoxia-inducible factor $1 \alpha$; IL, interleukin; PPAR $\gamma$, peroxisome proliferator-activated receptor $\gamma$; TFEB, transcription factor EB.

activation are known to counteract NLRP3 inflammasome activity ${ }^{15}$ and mediate HIF- $1 \alpha$ degradation. ${ }^{16}$ PI3K is required for autophagy and its inhibitor LY294002 can inhibit autophagic sequestration, a required first step of autophagy. ${ }^{31}$ Therefore, we aimed to examine if autophagy inhibition by LY294002 could negate the 

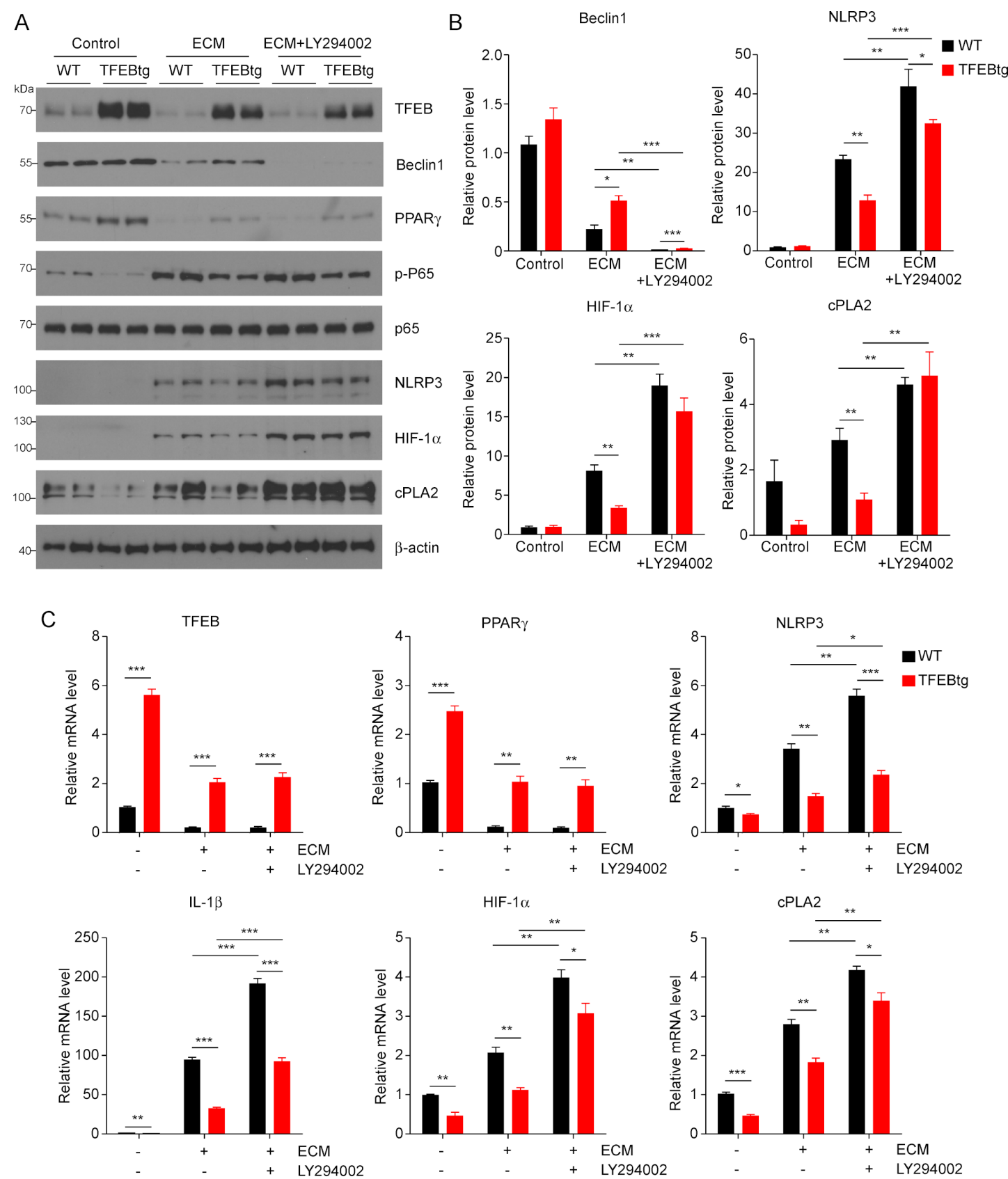

Figure 4 Autophagy-lysosome axis partially mediates the effects of transcription factor EB (TFEB) in macrophages (MФs). (A) Western blot analysis of indicated proteins in wild-type (WT) or TFEB transgenic (TFEBtg) bone marrow-derived MФs (BMDMs) treated with EO771 cell conditioned medium (ECM) with or without LY294002, a PI3K and autophagy inhibitor. (B) Band intensities of selected proteins were quantified by Gel-Pro Analyzer software and normalized by $\beta$-actin protein; $n=3,{ }^{*} p<0.05$, ${ }^{* *} p<0.01$. The quantification of other proteins is shown in online supplementary figure S4. (C) Quantitativereal-time PCR analysis of the expression of indicated genes in WT or TFEBtg BMDMs treated with ECM with or without LY294002. Data are presented as mean $\pm S D ; n=3$. ${ }^{*} p<0.05,{ }^{* *} \mathrm{p}<0.01,{ }^{* \star *} \mathrm{p}<0.001$; two-tailed Student's t-test. cPLA2, cytosolic phospholipases A2; HIF-1 $\alpha$, hypoxia-inducible factor $1 \alpha$; IL, interleukin; NLRP3, NLR Family Pyrin Domain Containing 3; PPAR $\gamma$, peroxisome proliferatoractivated receptor $\gamma$.

impact of TFEB overexpression on MФs. We treated WT and TFEBtg BMDMs with ECM with or without LY294002 and measured the expression of some of the key molecules mentioned previously. LY294002 could effectively block autophagy by abolishing Beclin 1 protein, but it did not change the expression of TFEB and PPAR $\gamma$, nor did it affect p65 phosphorylation in either ECMtreated WT or TFEBtg BMDMs (figure 4A-C and online supplementary figure S4). Importantly, autophagy inhibition significantly enhanced NLRP3, IL-1 $\beta$, HIF-1 $\alpha$, and cPLA2 expression in both ECM-treated WT and TFEBtg BMDMs, and diminished the differences in HIF- $1 \alpha$ and cPLA2 protein levels in those cells (figure 4A-C and online supplementary figure S4). These results suggest that autophagy partially mediates the beneficial effects of TFEB on TAMs. 


\section{Trehalose activates MФ TFEB and suppresses orthotopic breast tumor growth}

Our previous study showed that trehalose, a natural disaccharide, activates MФ TFEB and enhances MФ autophagy and phagocytosis of apoptotic cells. ${ }^{21}$ We here first confirmed that similar to HP $\beta C D$, also a TFEB activator, ${ }^{32}$ and chloroquine, an autophagic flux inhibitor that activates TFEB ${ }^{33}$ trehalose increased TFEB nuclear translocation and total cellular TFEB in ECM-treated MФs (online supplementary figure S5A,B). To test whether trehalose can modulate TAM function in breast tumors through activating TFEB, we treated BMDMs with ECM with or without trehalose. qPCR showed that while trehalose did not affect the expression of TFEB in ECMtreated MФs, activation of TFEB by trehalose replicated the gene expression profile observed in TFEBtg MФs, including increased expression of PPAR $\gamma$ and Lamp1, and decreased expression of NLRP3, IL-1 $\beta$, HIF-1 $\alpha$, cPLA2 and COX2 (figure 5A). The results were further confirmed by western blot analysis (figure $5 \mathrm{~B}$ and online supplementary figure S5C). Furthermore, trehalose treatment reduced the secretion of IL-1 $\beta$, IL-6, and PGE2 from ECM-treated MФs (figure 5C). We then examined if the activation of TFEB by trehalose is dependent on autophagy. We treated BMDMs from MФ-specific Atg5 knockout mice ${ }^{25}$ and control WT littermates with trehalose, and we found that (1) Atg5 depletion is very efficient in the MФs from MФ-specific Atg5 knockout mice, (2) trehalose significantly increased Atg5 expression in WT MФs but not in Atg5-deficient MФs, and (3) trehalose equally increased the expression of TFEB targeted gene PPAR $\gamma$ in WT and Atg5-deficient MФs (online supplementary figure S5D). These results confirmed that trehalose activates TFEB in MФs independent of autophagy since Atg5 is required for autophagy.

We next tested if trehalose treatment could inhibit breast tumor growth. We treated female Balb/c mice with trehalose for 25 days at $2 \mathrm{~g} / \mathrm{kg}$, three times per week, starting 1 day after $4 \mathrm{~T} 1$ cell inoculation, and monitored the growth of the tumors. It is shown that trehalose treatment significantly delayed tumor growth (figure 5D). To verify if the benefit of trehalose in indeed due to the modulation of the immune microenvironment, we performed qPCR to measure the expression of Arg1 and IFN $\gamma$ to reflect the abundance of M2-like TAMs and cytotoxic $\mathrm{T}$ cells, respectively, in the tumors. We found that the tumors from trehalose treated mice displayed significantly lower expression of Arg1 but higher expression of IFN $\gamma$ (online supplementary figure S6A). The experiment was repeated using $\mathrm{C} 57 \mathrm{Bl} / 6$ mice inoculated with EO771 cells; a sucrose control group also was included. The results show that trehalose, but not sucrose, significantly reduced breast tumor growth (figure 5E). In addition, we used the C3(1)/SV40Tag spontaneous breast tumor mouse model to confirm the effects of trehalose. Female C3(1)/SV40Tag mice were treated with trehalose $(2 \mathrm{~g} / \mathrm{kg}$, three times a week) or vehicle from 6 to 17 weeks of age. The results demonstrated a trend in the reduction of mammary gland tumor size related to trehalose administration but not in the number of tumors (online supplementary figure S6B).

\section{TFEB expression is a positive marker for breast cancer prognosis}

We used the TCGA database to analyze the association between the expression of TFEB or its regulated genes and breast cancer survival. We found 822 patients with low TFEB expression and 253 patients with high TFEB expression. The data indicate that 5 -year survival is significantly higher in the patients with higher TFEB expression levels compared with those with lower TFEB expression levels ( $87 \%$ vs $80 \%, \mathrm{p}=0.0036$ ) (figure $6 \mathrm{~A}$ ). Similarly, higher expression levels of SOCS3 and PPAR $\gamma$ are correlated with a higher survival rate, while higher expression levels of HIF-1 $\alpha$ are associated with a lower survival rate (figure 6A). The result was confirmed using another online analysis platform. TFEB, SOCS3, and PPAR $\gamma$ are shown to be positive prognostic factors, while HIF- $1 \alpha$ is a negative prognostic factor (figure $6 \mathrm{~B}$ ). Although the expression of these genes is not only from TAMs, the data suggest that activating TFEB and thus increasing the expression of SOCS3 and PPAR $\gamma$ while reducing the expression of HIF- $1 \alpha$ in breast tumors may be beneficial for patients with breast cancer. To further examine if suppression of TFEB expression in TAMs is true in human breast tumors, we stained TFEB using human breast tumor specimens. Breast tumors from five triple negative breast cancer patients were stained for CD68, TFEB and 4',6-diamidino-2-phenylindole (DAPI). The data show that TFEB staining intensity is significantly lower in the MФs in the tumor nest than that in the tumor periphery (online supplementary figure $\mathrm{S} 7$ ).

\section{DISCUSSION}

As high as $50 \%$ of the cells within breast tumors are МФs, called TAMs; and these TAMs play important roles in tumor development, metastasis, and resistance to therapies including recently developed immune check point inhibitors. ${ }^{34}$ Therefore, targeting TAMs is an actively pursued strategy for solid tumor treatment. МФ depletion is not a viable approach since these cells are the first line of defense against infection and play vital roles in many physiological processes. Blocking the tumor-promoting signaling pathways and antagonizing resulting key effector molecules in TAMs are promising approaches. However, there are many interconnected pathways that collectively define the phenotype of TAMs and numerous effector molecules that execute tumor-promoting functions, thus targeting any single pathway or effector molecule is not sufficient to achieve clinical benefit.

We identified TFEB as a target whose activation results in beneficial alterations in several key pathways and suppression of many tumor-promoting effector molecules in TAMs (figure 6C). First, TFEB upregulates SOCS3 and thus suppresses STAT3 activation, thereby blocking 

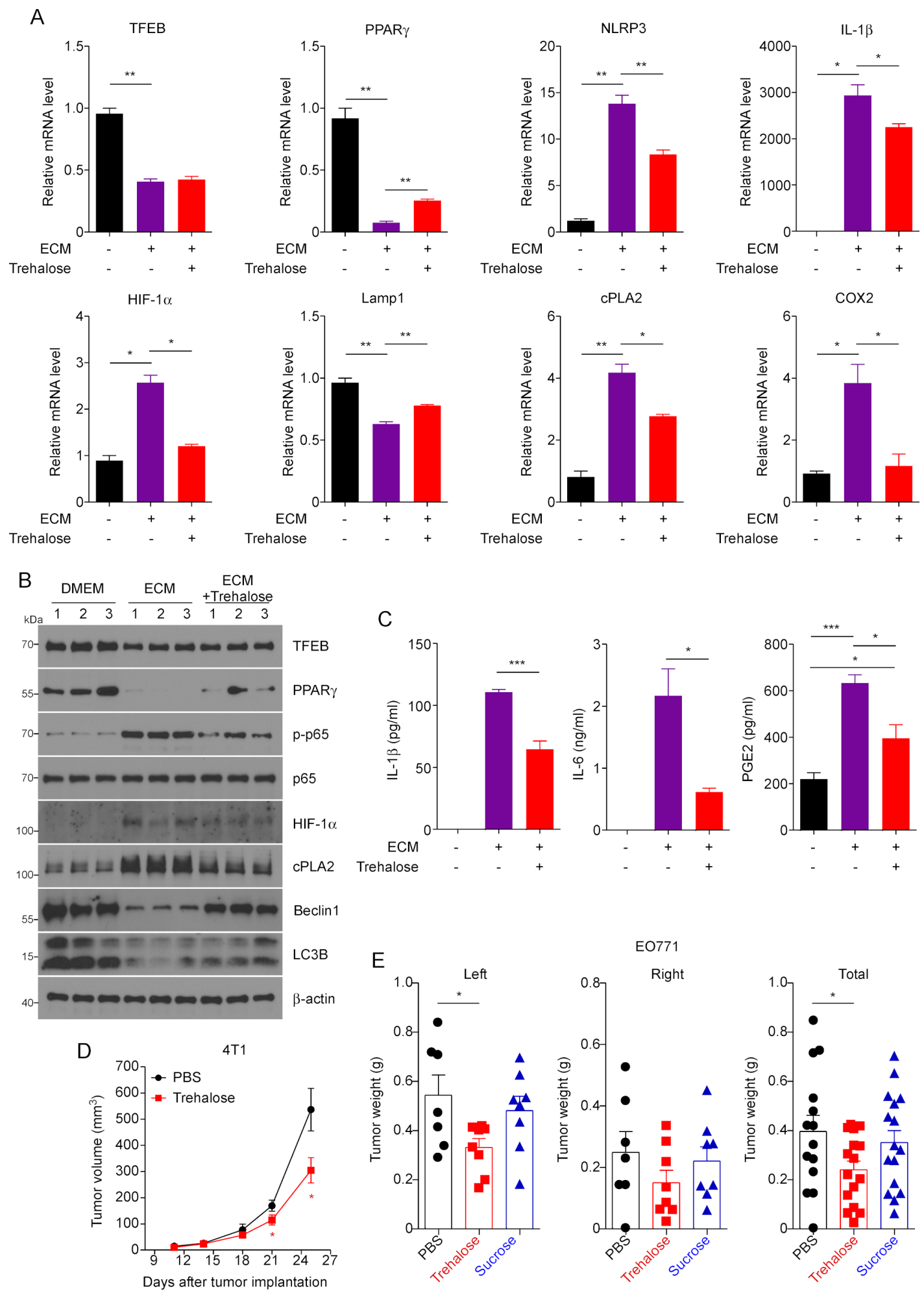

Figure 5 TFEB activator trehalose modulates macrophages (MФs) function and suppresses breast tumor growth. (A) Quantitative real-time PCR analysis of expression of the indicated genes in mouse peritoneal MФs (pMФs) treated EO771 cell conditioned medium (ECM) with or without trehalose. (B) Western blot analysis of indicated proteins in mouse pMФs treated with ECM with or without trehalose. The quantification is shown in online supplementary figure S5C. (C) IL-1 $\beta$, IL-6 and PGE2 productions were determined in medium of mouse $\mathrm{pM \Phi s} \mathrm{treated} \mathrm{with} \mathrm{ECM}$ with or without trehalose. $\mathrm{n}=4,{ }^{*} \mathrm{p}<0.05,{ }^{* *} \mathrm{p}<0.01$. (D) Growth curve of orthotopic 4T1 breast tumors in female Balb/c mice treated with vehicle or trehalose. Data are expressed as mean \pm SD for each group; Student's t-test; ${ }^{*} p<0.05$, vs Veh (vehicle). (E) EO771 breast tumor-bearing mice were treated with Veh, trehalose or sucrose for 20 days before the mice were sacrificed for tumor measurement. One-way analysis of variance with Bonferroni's post-test; ${ }^{*} p<0.05$. COX2, cyclo-oxygenase-2; cPLA2, cytosolic phospholipases A2; HIF-1 $\alpha$, hypoxia-inducible factor $1 \alpha$; IL, interleukin; MФs, macrophages; NLRP3, NLR Family Pyrin Domain Containing 3; PBS, phosphate-buffered saline; PGE, prostaglandin E2; PPAR $\gamma$, peroxisome proliferator-activated receptor $\gamma$; TFEB, transcription factor EB. 
A
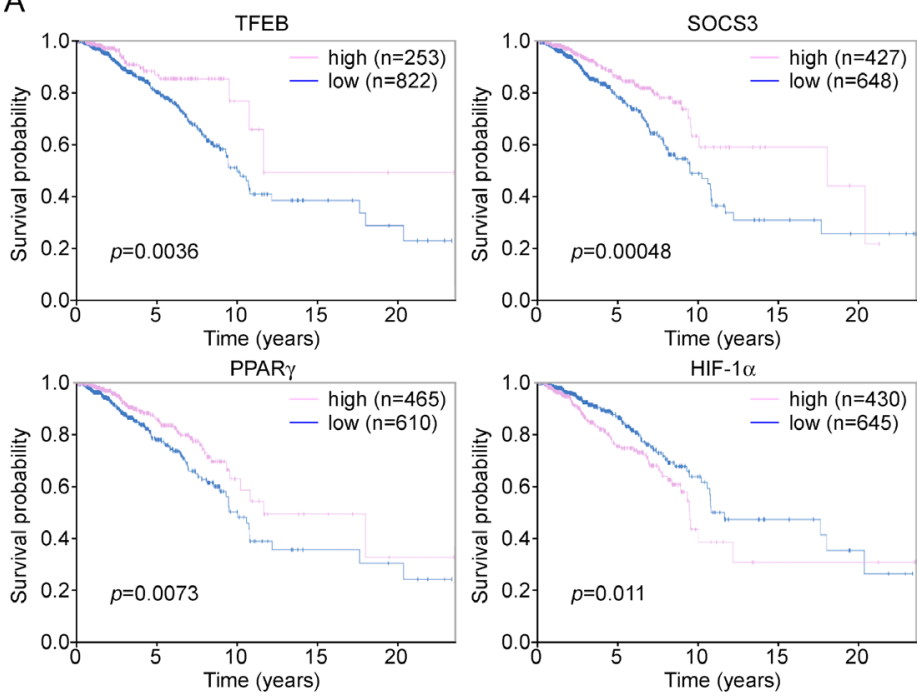

HIF- $1 \alpha$

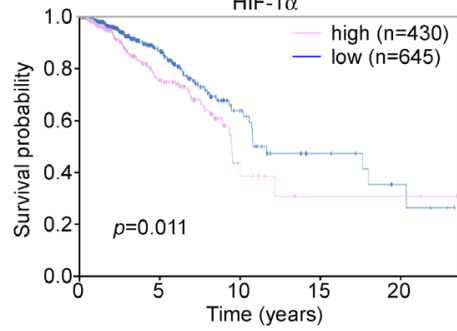

B

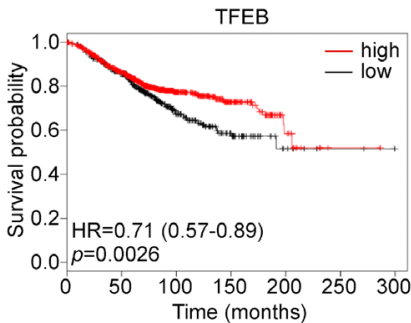

PPARY

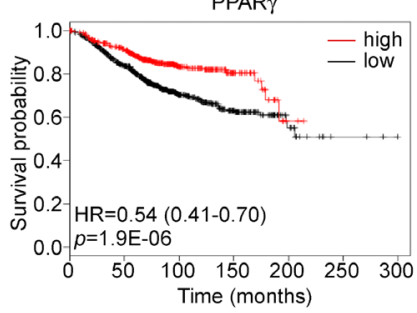

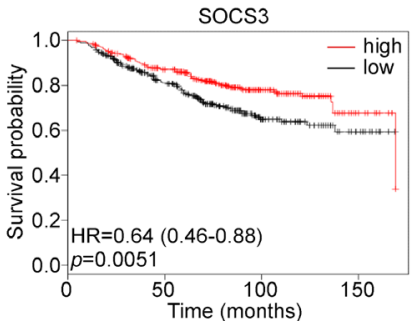

HIF-10

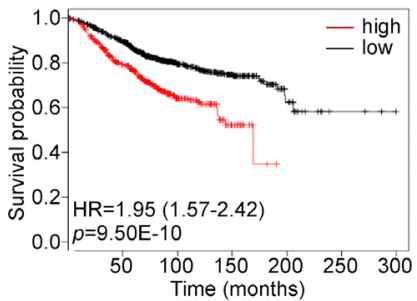

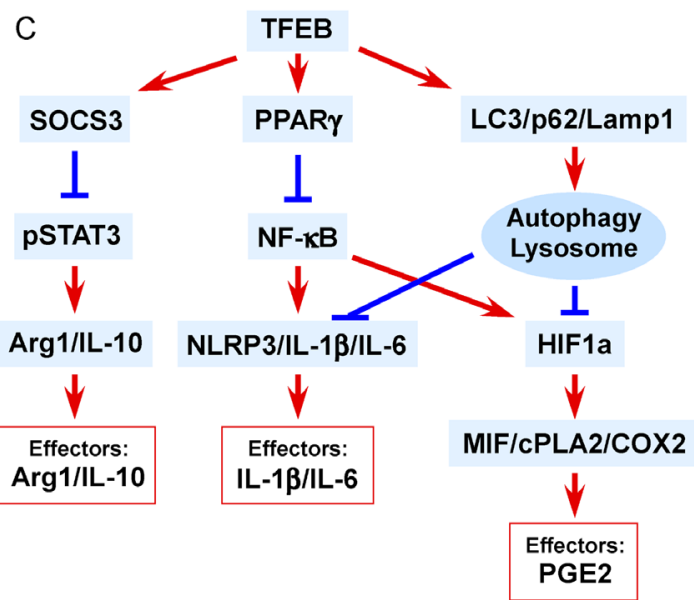

Figure 6 The clinical relevance of macrophage (MФ) signaling axes regulated by transcription factor EB (TFEB). (A) The prognostic value of TFEB and the genes regulated by TFEB for patients with breast cancer based on the CTGA Human Protein Atlas database. (B) The prognostic value of TFEB and the genes regulated by TFEB for patients with breast cancer based on the online survival analysis platform www.kmplot.com. (C) A schematic drawing depicting how TFEB regulates macrophage functions in breast tumors. Arg1, arginase 1; COX2, cyclo-oxygenase-2; cPLA2, cytosolic phospholipases A2; HIF-1 $\alpha$, hypoxiainducible factor $1 \alpha$; IL, interleukin; NLRP3, NLR Family Pyrin Domain Containing 3; PGE, prostaglandin E2; PPAR $\gamma$, peroxisome proliferator-activated receptor $\gamma$; SOCS3, suppressor of cytokine signaling 3. 
MФ M2-like polarization and the production of Arg1 and IL-10. ${ }^{23}$ Second, TFEB activates the transcription of PPAR $\gamma$, which blunts NFKB activation, resulting in downregulation of NLRP3/IL-1 $\beta /$ IL-6 axis and HIF- $1 \alpha$ transcription. Third, TFEB enhances autophagy and lysosome activities, which counteract the NLRP3 inflammasome as well as lead to degradation of HIF-1 $\alpha$ protein.

PPAR $\gamma$ is at the critical crossroad of inflammation and lipid metabolism. In TAMs, NFKB is activated through multiple pathways, including TLR activation ${ }^{35}$ and response to hypoxia, ${ }^{36}$ leading to transcription of proinflammatory cytokines and HIF- $1 \alpha .{ }^{37}$ PPAR $\gamma$ acts as an E3 ligase that induces the degradation of $\mathrm{NF \kappa B} / \mathrm{p} 65 .{ }^{37}$ Our data demonstrate that TFEB upregulates PPAR $\gamma$ by directly binding to its promoter, and thereby downregulates inflammatory mediators NLRP3, IL-1 $\beta$ and IL6, and HIF-1 $\alpha$.

During MФ inflammatory responses, NLRP3 and IL-1 $\beta$ expression is controlled by NF-kB (nuclear factor kappalight-chain-enhancer of activated B cells), and NLRP3 inflammasome processes pro-IL-1 $\beta$ protein into mature IL-1 $\beta .^{38}$ In breast cancer, IL- $1 \beta$ has been linked to the proliferation, invasion, angiogenesis, and inhibition of apoptosis in cancer cells. ${ }^{39}$ IL-1 $\beta$ further induces IL-6 expression $^{40}$; and IL-6 not only induces cancer cell proliferation, but also induces EMT and the formation and maintenance of breast cancer stem cells. ${ }^{41}$ The NLRP3 inflammasome is known to be negatively regulated by autophagy/lysosome. ${ }^{15}$ Our data show that in TAMs, TFEB may negatively regulate NLRP3 inflammasome via PPAR $\gamma$-mediated NFאB suppression and enhanced autophagy and lysosome activities.

In response to the hypoxic TME, the expression of HIF-1 $\alpha$ is upregulated and the protein is stabilized in cancer cells as well as stromal cells including TAMs. Among several downstream signaling events, HIF-1 $\alpha$ in MФs activates the MIF/cPLA2/COX2 axis, leading to PGE2 production. ${ }^{13}$ PGE2 promotes breast cancer progression by multiple mechanisms: inactivation of host antitumor immune cells, ${ }^{42}{ }^{43}$ stimulation of tumor cell migration and invasiveness, ${ }^{44-46}$ induction of stem-like cells, ${ }^{47}$ tumor-associated angiogenesis ${ }^{44}$ and lymphangiogenesis. ${ }^{48}$ Our results suggest that in breast tumors, TFEB suppresses HIF- $1 \alpha$ transcription via PPAR $\gamma$-mediated NFKB inactivation and also reduces HIF- $1 \alpha$ protein by autophagy/lysosome-mediated degradation in TAMs, leading to suppression of the MIF/cPLA2/COX2 signaling pathway and reduced production of PGE2.

These cell culture study results are further strengthened by mouse studies showing that (1) MФ-specific TFEB knockout promoted breast tumor growth by enhancing the tumor-promoting phenotype of TAMs; and (2) trehalose, a TFEB activator, modulated MФ gene expression and function towards a less tumor-promoting phenotype and thereby inhibited breast tumor growth in multiple breast cancer mouse models. Moreover, analysis of breast cancer patient tumor genome data revealed that TFEB, SOCS3, and PPAR $\gamma$ are positive prognostic markers of breast cancer, while HIF- $1 \alpha$ is a negative prognostic marker. While TAMs only contribute to part of the expression levels of these genes, the human data suggest that therapies that increase the expression or activity of TFEB in TAMs as well as other cell types in breast tumors may be effective in halting breast cancer development.

\section{CONCLUSIONS}

Our study identifies TFEB as a master regulator of TAMs in breast cancer. TFEB controls TAM gene expression and function through multiple pathways. By activating SOCS3, TFEB suppresses STAT3 signaling for M2-like activation of MФs. By upregulating PPAR $\gamma$, TFEB suppresses NF-кB activation and thus inflammasome activity and HIF- $1 \alpha$ mediated hypoxic response. By activating autophagy/lysosome activities, TFEB promotes degradation of NLRP3 inflammasomes and HIF-1 $\alpha$ protein. Collectively, TFEB activation simultaneously inhibits multiple effector molecules that exert the tumor-promoting function of TAMs, including Arg1, IL-10, IL-1 $\beta$, IL-6 and PGE2. Therefore, TFEB is a promising therapeutic target for breast cancer and possibly other solid malignant tumors.

\section{Author affiliations}

${ }^{1}$ Department of Cell Biology and Anatomy, University of South Carolina School of Medicine, Columbia, South Carolina, USA

${ }^{2}$ Cardiovascular Division, Department of Medicine and Department of Pathology \& Immunology, Washington University in Saint Louis, Saint Louis, Missouri, USA

${ }^{3}$ Department of Drug Discovery and Biomedical Sciences, University of South Carolina College of Pharmacy, Columbia, South Carolina, USA

${ }^{4}$ Department of General Surgery, Nanjing Drum Tower Hospital, the Affiliated Hospital of Nanjing University Medicine School, Nanjing, China

${ }^{5}$ Department of Pathology, Microbiology and Immunology, University of South Carolina School of Medicine, Columbia, South Carolina, USA

Contributors $\mathrm{DF}$ and $\mathrm{JH}$ designed the study; $\mathrm{YL}$ and $\mathrm{JH}$ performed most of the experiments; QL, JW and YW assisted in the experiments; TDE and BR generated the M $\Phi$-TFEBtg and M $\Phi$-TFEB-/- mice; EAM provided the C3(1)/SV40Tag. DA performed the microarrays. DF and YL drafted the manuscript; JH, YY, BR, and EAM helped in data interpretation and manuscript writing. All authors read and approved the final manuscript.

Funding This work was supported by the National Institute of Health grants R01CA218578 (to DF and EAM) and R21CA216230 (to DF). JH has been supported by the Susan G. Komen trainee program (GTDR17500160).

Competing interests None declared.

Patient consent for publication Not required.

Ethics approval Animal care procedures and experimental methods were approved by the Institutional Animal Care and Use Committee of the University of South Carolina according to National Institutes of Health guidelines.

Provenance and peer review Not commissioned; externally peer reviewed.

Data availability statement Data are available in a public, open access repository. The microarray data have been deposited in Gene Expression Omnibus (GEO) database (accession numbers GSE73311 and GSE139554).

Open access This is an open access article distributed in accordance with the Creative Commons Attribution Non Commercial (CC BY-NC 4.0) license, which permits others to distribute, remix, adapt, build upon this work non-commercially, and license their derivative works on different terms, provided the original work is properly cited, appropriate credit is given, any changes made indicated, and the use is non-commercial. See http://creativecommons.org/licenses/by-nc/4.0/. 


\section{ORCID iD}

Daping Fan http://orcid.org/0000-0002-4729-761X

\section{REFERENCES}

1 Salmaninejad A, Valilou SF, Soltani A, et al. Tumor-associated macrophages: role in cancer development and therapeutic implications. Cell Oncol 2019;42:591-608.

2 Mantovani A, Marchesi F, Malesci A, et al. Tumour-associated macrophages as treatment targets in oncology. Nat Rev Clin Oncol 2017;14:399-416.

3 Lin Y, Xu J, Lan H. Tumor-associated macrophages in tumor metastasis: biological roles and clinical therapeutic applications. $\checkmark$ Hematol Oncol 2019;12:76.

4 Murdoch C, Giannoudis A, Lewis CE. Mechanisms regulating the recruitment of macrophages into hypoxic areas of tumors and other ischemic tissues. Blood 2004;104:2224-34.

5 Swierczak A, Cook AD, Lenzo JC, et al. The promotion of breast cancer metastasis caused by inhibition of CSF-1R/CSF-1 signaling is blocked by targeting the G-CSF receptor. Cancer Immunol Res 2014;2:765-76.

6 Rodriguez PC, Quiceno DG, Zabaleta J, et al. Arginase I production in the tumor microenvironment by mature myeloid cells inhibits T-cell receptor expression and antigen-specific T-cell responses. Cancer Res 2004;64:5839-49.

7 Smith LK, Boukhaled GM, Condotta SA, et al. Interleukin-10 Directly Inhibits $\mathrm{CD}^{+} \mathrm{T}$ Cell Function by Enhancing N-Glycan Branching to Decrease Antigen Sensitivity. Immunity 2018;48:299-312.

8 Colak S, Ten Dijke P. Targeting TGF- $\beta$ signaling in cancer. Trends Cancer 2017;3:56-71.

9 Landskron G, De la Fuente M, Thuwajit P, et al. Chronic inflammation and cytokines in the tumor microenvironment. J Immunol Res 2014;2014:1-19.

10 Baker KJ, Houston A, Brint E. IL-1 family members in cancer; two sides to every story. Front Immunol 2019;10:1197.

11 Petrova V, Annicchiarico-Petruzzelli M, Melino G, et al. The hypoxic tumour microenvironment. Oncogenesis 2018;7:10.

12 Chen EP, Smyth EM. COX-2 and PGE2-dependent immunomodulation in breast cancer. Prostaglandins Other Lipid Mediat 2011;96:14-20.

13 Nandi P, Girish GV, Majumder M, et al. PGE2 promotes breast cancer-associated lymphangiogenesis by activation of EP4 receptor on lymphatic endothelial cells. BMC Cancer 2017;17:11

14 Mathew R, Karantza-Wadsworth V, White E. Role of autophagy in cancer. Nat Rev Cancer 2007;7:961-7.

15 Saitoh T, Akira S. Regulation of inflammasomes by autophagy. J Allergy Clin Immunol 2016;138:28-36.

16 DePavia A, Jonasch E, Liu X-D. Autophagy degrades hypoxia inducible factors. Mol Cell Oncol 2016;3:e1104428.

17 Settembre C, Di Malta C, Polito VA, et al. TFEB links autophagy to lysosomal biogenesis. Science 2011;332:1429-33.

18 Settembre C, Medina DL. TFEB and the CLEAR network. Methods Cell Biol 2015;126:45-62.

19 Martini-Stoica H, Xu Y, Ballabio A, et al. The autophagy-lysosomal pathway in neurodegeneration: a TFEB perspective. Trends Neurosci 2016;39:221-34.

20 Napolitano G, Ballabio A. TFEB at a glance. J Cell Sci 2016;129:2475-81.

21 Sergin I, Evans TD, Zhang X, et al. Exploiting macrophage autophagy-lysosomal biogenesis as a therapy for atherosclerosis. Nat Commun 2017;8:15750.

22 Brady OA, Martina JA, Puertollano R. Emerging roles for TFEB in the immune response and inflammation. Autophagy 2018;14:181-9.

23 Fang L, Hodge J, Saaoud F, et al. Transcriptional factor EB regulates macrophage polarization in the tumor microenvironment. Oncoimmunology 2017;6:e1312042.

24 Settembre C, Zoncu R, Medina DL, et al. A lysosome-to-nucleus signalling mechanism senses and regulates the lysosome via mTOR and TFEB. EMBO J 2012;31:1095-108.

25 Emanuel R, Sergin I, Bhattacharya S, et al. Induction of lysosomal biogenesis in atherosclerotic macrophages can rescue lipid-induced lysosomal dysfunction and downstream sequelae. Arterioscler Thromb Vasc Biol 2014;34:1942-52.

26 Huang X, Li Y, Fu M, et al. Polarizing macrophages in vitro. Methods Mol Biol 2018:1784:119-26.

27 Györffy B, Lanczky A, Eklund AC, et al. An online survival analysis tool to rapidly assess the effect of 22,277 genes on breast cancer prognosis using microarray data of 1,809 patients. Breast Cancer Res Treat 2010;123:725-31.

28 Iwanowycz S, Wang J, Altomare D, et al. Emodin bidirectionally modulates macrophage polarization and epigenetically regulates macrophage memory. J Biol Chem 2016;291:11491-503.

29 Fisher DE, Carr CS, Parent LA, et al. TFEB has DNA-binding and oligomerization properties of a unique helix-loop-helix/leucine-zipper family. Genes Dev 1991;5:2342-52.

30 Salma N, Song JS, Kawakami A, et al. Tfe3 and Tfeb transcriptionally regulate peroxisome proliferator-activated receptor $\gamma 2$ expression in adipocytes and mediate adiponectin and glucose levels in mice. Mol Cell Biol 2017;37:e00608-16.

31 Blommaart EF, Krause U, Schellens JP, et al. The phosphatidylinositol 3-kinase inhibitors wortmannin and LY294002 inhibit autophagy in isolated rat hepatocytes. Eur J Biochem 1997;243:240-6.

32 Song W, Wang F, Lotfi P, et al. 2-Hydroxypropyl- $\beta$-cyclodextrin promotes transcription factor EB-mediated activation of autophagy: implications for therapy. J Biol Chem 2014;289:10211-22.

33 Chen D, Xie J, Fiskesund R, et al. Chloroquine modulates antitumor immune response by resetting tumor-associated macrophages toward M1 phenotype. Nat Commun 2018;9:873.

34 Qiu S-Q, Waaijer SJH, Zwager MC, et al. Tumor-associated macrophages in breast cancer: Innocent bystander or important player? Cancer Treat Rev 2018;70:178-89.

35 Mancino A, Lawrence T. Nuclear factor-kappaB and tumorassociated macrophages. Clin Cancer Res 2010;16:784-9.

36 D'Ignazio L, Rocha S, NF-kappaB HI. Hypoxia induced NF-кB. Cells 2016;5. doi:10.3390/cells5010010. [Epub ahead of print: 08 Mar 2016].

37 van Uden P, Kenneth NS, Rocha S. Regulation of hypoxia-inducible factor-1alpha by NF-kappaB. Biochem J 2008;412:477-84.

38 Liu T, Zhang L, Joo D, et al. NF-kappaB signaling in inflammation. Sig Transduct Target Ther 2017;2.

39 Tulotta C, Ottewell P. The role of IL-1B in breast cancer bone metastasis. Endocr Relat Cancer 2018;25:R421-34.

40 Oh K, Lee O-Y, Park Y, et al. IL-1 $\beta$ induces IL-6 production and increases invasiveness and estrogen-independent growth in a TG2-dependent manner in human breast cancer cells. BMC Cancer 2016;16:724

41 Dethlefsen C, Højfeldt G, Hojman P. The role of intratumoral and systemic IL-6 in breast cancer. Breast Cancer Res Treat 2013;138:657-64.

42 Lala PK, Parhar RS, Singh P. Indomethacin therapy abrogates the prostaglandin-mediated suppression of natural killer activity in tumor-bearing mice and prevents tumor metastasis. Cell Immunol 1986;99:108-18.

43 Lala PK, Al-Mutter N, Orucevic A. Effects of chronic indomethacin therapy on the development and progression of spontaneous mammary tumors in C3H/HEJ mice. Int J Cancer 1997;73:371-80.

44 Rozic JG, Chakraborty C, Lala PK. Cyclooxygenase inhibitors retard murine mammary tumor progression by reducing tumor cell migration, invasiveness and angiogenesis. Int $J$ Cancer 2001:93:497-506.

45 Timoshenko AV, Xu G, Chakrabarti S, et al. Role of prostaglandin E2 receptors in migration of murine and human breast cancer cells. Exp Cell Res 2003;289:265-74.

46 Timoshenko AV, Lala PK, Chakraborty C. PGE2-mediated upregulation of iNOS in murine breast cancer cells through the activation of EP4 receptors. Int J Cancer 2004;108:384-9.

47 Majumder M, Xin X, Liu L, et al. Prostaglandin E2 receptor EP4 as the common target on cancer cells and macrophages to abolish angiogenesis, lymphangiogenesis, metastasis, and stem-like cell functions. Cancer Sci 2014;105:1142-51.

48 Timoshenko AV, Chakraborty C, Wagner GF, et al. COX-2-mediated stimulation of the lymphangiogenic factor VEGF-C in human breast cancer. Br J Cancer 2006;94:1154-63. 